\title{
Testing constraints on rRNA bases that make nonsequence-specific contacts with the codon.anticodon complex in the ribosomal A site
}

\author{
DWAYNE L. TALIAFERRO and PHILIP J. FARABAUGH \\ Program in Molecular and Cell Biology, Department of Biological Sciences, University of Maryland, Baltimore County, Baltimore, MD 21250, USA
}

\begin{abstract}
During protein synthesis, interactions between the decoding center of the ribosome and the codon-anticodon complexes maintain translation accuracy. Correct aminoacyl-tRNAs induce the ribosome to shift into a "closed" conformation that both blocks tRNA dissociation and accelerates the process of tRNA acceptance. As part of the ribosomal recognition of cognate tRNAs, the rRNA nucleotides G530 and A1492 form a hydrogen-bonded pair that interacts with the middle position of the codon-anticodon complex and recognizes correct Watson-Crick base pairs. Exchanging these two nucleotides (A530 and G1492) would not disrupt these interactions, suggesting that such a double mutant ribosome might properly recognize tRNAs and support viability. We find, however, that exchange mutants retain little ribosomal activity. We suggest that even though the exchanged nucleotides might function properly during tRNA recruitment, they might disrupt one or more other functions of the nucleotides during other stages of protein synthesis.
\end{abstract}

Keywords: accuracy center; dedicated ribosome; rRNA mutations; tRNA recognition

\section{INTRODUCTION}

Translation of genetic information encoded in nucleic acids into proteins occurs on the ribosome, a molecular machine that orchestrates the interaction between the mRNA, tRNAs, and translation factors to allow rapid and accurate protein synthesis. Accuracy depends on efficiently accepting correct (cognate) aminoacyl-tRNAs and rejecting incorrect ones (near cognate or noncognate). For 30 years the prevailing model for the maintenance of translational accuracy was kinetic proofreading (Hopfield 1974; Ninio 1975), which hypothesized that ribosomes use the intrinsic instability of incorrect aminoacyl-tRNA.mRNA complexes to select against them in a two-stage discrimination reaction (Thompson 1988; Rodnina et al. 2005). Aminoacyl-tRNAs bind to the ribosomal A site in a ternary complex with elongation factor $1 \mathrm{~A}$ (EF-1A) and GTP. The model proposed that incorrect ternary complexes dissociated much more rapidly than do cognate complexes in the first stage of discrimination that occurs before

Reprint requests to: Philip J. Farabaugh, Program in Molecular and Cell Biology, Department of Biological Sciences, University of Maryland, Baltimore County, Baltimore, MD 21250, USA; e-mail: farabaug@umbc. edu; fax: (410) 455-3875.

Article published online ahead of print. Article and publication date are at http://www.rnajournal.org/cgi/doi/10.1261/rna.552007. hydrolysis of the GTP. In the second discrimination stage after GTP hydrolysis, incorrect aminoacyl-tRNAs again tend to dissociate from the ribosome.EF1A.GDP complex while correct ones remain bound. Under this model, these effects result from a difference in the binding rate constants for correct and incorrect complexes; a 100-fold preference for the cognate complex at each stage compounds to provide the $\sim 10,000$-fold preference (Thompson 1988).

More recent work shows that these thermodynamic effects cannot explain the observed accuracy (Rodnina et al. 2005). Instead, the most critical parameter in discrimination actually is the rate of activation of the intrinsic GTPase of EF-1A. This rate is almost three orders of magnitude faster for correct complexes. The ribosome cannot maximize discrimination using stability differences because it proceeds to the next step in the reaction too rapidly to allow the full thermodynamic difference to be achieved (Gromadski and Rodnina 2004).

Correct and incorrect ternary complexes associate with the ribosome equally rapidly, but a correct codon-anticodon complex forms a web of interactions with nucleotides in the rRNA and portions of the ribosomal protein $\mathrm{rpS} 12$ (Ogle et al. 2001, 2002). These contacts require the geometry of Watson-Crick base pairs, which do not form when a near-cognate codon binds the A site (Ogle et al. 2002). Our current model of discrimination argues that 
interactions with the cognate codon-anticodon complex both stabilize correct complexes and cause the large-scale ribosomal rearrangement that ultimately increases the rate of GTPase activation because a bound cognate tRNA induces a change in ribosome structure. This large-scale ribosomal rearrangement both stabilizes the complexes on the ribosome and increases the rate of activation of the EF1A GTPase (Rodnina et al. 2005).

One method to analyze the mechanism of discrimination is through computer modeling starting from unsolved ribosomal structures. Early approaches to address this problem involved docking of static molecular models (VanLoock et al. 1999; Lim and Curran 2001; Simonson and Lake 2002). A more powerful approach, molecular dynamics (Karplus and McCammon 2002), uses classical Newtonian mechanics to calculate the movement of atoms in a determined structure under the influence of interatomic forces and heat. This powerful technique has been used to successfully model the dynamics of biologically relevant macromolecular complexes (Karplus and McCammon 2002). Analyzing large complexes can be very computationally expensive. Despite this limitation, molecular dynamics studies attempting to predict the behavior of prokaryotic ribosomes have successfully addressed the relative stability of codon-anticodon complexes formed by correct (cognate) and incorrect (near cognate and noncognate) anticodons (Sanbonmatsu and Joseph 2003). The study suggested that discrimination depended on the stability of base pairing and on the geometry of the hydrogen bonds formed. A high-resolution structure of the Thermus thermophilus 30S subunit with a cognate codon-anticodon complex (Ogle et al. 2001) shows bridging contacts to the two 2 ' hydroxyls of the first and second base pairs by rRNA nucleotide A1493 and the G530-A1492 pair, respectively. These interactions depend on the Watson-Crick geometry of the base interaction, which noncognate pairs do not have. The simulations by Sanbonmatsu and Joseph (2003) showed that even middle position codon-anticodon mismatches that formed stable pairs failed to interact with G530-A1492. On the other hand, the simulations suggested that although A1493 stabilizes first position base pairs in cognate interactions, it actually destabilizes near-cognate pairing. This suggests that the ribosome uses distinct mechanisms to distinguish correct and incorrect pairing at the two codon positions.

Sanbonmatsu and Joseph (2003) noted that the G530A1492 bridging interaction used hydrogen bonding from $\mathrm{N} 4$ of G530 to the $2^{\prime}-\mathrm{OH}$ of the anticodon nucleotide and from N4 of A1492 to the 2'-OH of the codon nucleotide. They realized that exchanging bases at 530 and 1492 (G530A and A1492G) would not disturb the geometry of this interaction. They predicted that a mutant ribosome with such an exchange mutation should be functional although each single mutant ribosome is inactive (Powers and Noller 1990; Santer et al. 1993; Yoshizawa et al. 1999;
Abdi and Fredrick 2005). We decided to test this prediction using a dedicated ribosome system (Lee et al. 1997). In this system, Escherichia coli cells express a 16S rRNA in which an alternative sequence replaces the normal Shine-Dalgarno interaction site (anti-SD). The anti-SD base-pairs with an mRNA site (SD) immediately upstream of initiation codons, directing the ribosome to initiation sites (Shine and Dalgarno 1974). Mutant ribosomes only translate mRNAs having a complementary alternative SD site and do not translate cellular mRNAs and endogenous ribosomes do not recognize the alternative SD site (Lee et al. 1997). This system allows otherwise lethal rRNA mutations to be tested without compromising the viability of the cell. We present our data showing that, contrary to the prediction, exchanging the G530 and A1492 residues does not result in a functional ribosome. We will discuss the implications for this result in terms of the presumed function of these rRNA residues during multiple steps of translation.

\section{RESULTS}

\section{Mutations to critical residues G530 and A1492 greatly reduce ribosomal activity in vivo}

Solved structures of prokaryotic ribosomes carrying tRNA anticodon stem-loops (ASLs) bound in the A site to cognate codons on model mRNAs show that 16S rRNA nucleotides G530 and A1492 directly interact with the middle base pair of the anticodon-codon complex. Any change to G530 or A1492 of the $16 \mathrm{~S}$ rRNA confers a dominant lethal phenotype in E. coli (Powers and Noller 1990; Santer et al. 1993; Yoshizawa et al. 1999; Abdi and Fredrick 2005). These mutant rRNAs incorporate into $30 \mathrm{~S}$ and 70S ribosomes (Powers and Noller 1990; Santer et al. 1993; Abdi and Fredrick 2005) and assemble into polysomes (Powers and Noller 1990), but they translate natural mRNAs significantly less efficiently than do wild-type ribosomes (Santer et al. 1993).

To quantify the in vivo activity of mutant ribosomes without affecting cell viability, we have used a modification of the dedicated ribosome system developed by Lee et al. (1997). The system employs mutant ribosomes in which the normal SD interaction site of $16 \mathrm{~S}$ rRNA, $5^{\prime}$-CCUCCU-3' is replaced by an alternative sequence, $5^{\prime}$-GGGAUU- $3^{\prime}$. These mutant ribosomes fail to recognize the canonical SD site, $5^{\prime}$ AGGAGG-3' but efficiently recognize an alternative SD site, $5^{\prime}$-AUCCC-3'. Endogenous ribosomes fail to recognize the alternative SD site but continue to recognize cellular genes with the normal SD site. A plasmid pDT10 encodes the alternative $16 \mathrm{~S}$ rRNA, under the control of an IPTGinducible lac promoter. Using QuikChange site-directed mutagenesis (Stratagene), we introduced all possible substitutions and the one-base deletion at either G530 or A1492.

To assay the function of the mutant ribosomes we introduced them into an E. coli strain (DT1) encoding a 
single copy of the lac $Z$ gene integrated ectopically at the atth site. In this gene, the alternative SD site replaces the normal SD so that it is translated only by the alternative ("dedicated") ribosomes (see Materials and Methods for a description of this construction). The pDT10 plasmids encoding wild-type and mutant dedicated 16S rRNAs were introduced into DT1 and assayed for expression of the lac $Z$ product, $\beta$-galactosidase using the standard procedure (Miller 1972). Expression of $\beta$-galactosidase requires the dedicated ribosome; the activity in the presence of the dedicated ribosomes (2000 \pm 20 units) was 200 times that in their absence (10 \pm 0.9 units). This result implies that no more than $0.5 \%$ of the protein product synthesized in the presence of the dedicated ribosomes resulted from translation by the endogenous ribosomes.

We first tested the activity of $\beta$-galactosidase translated by dedicated ribosomes carrying the mutations G530A, G530C, G530U, G530د (a 1 nucleotide [nt] deletion), A1492C, A1492G, A1492U, and A1492A. Each of the mutant ribosomes produced much less enzyme activity than wild type, with expression averaging $<2 \%$ the level of wild-type ribosomes (Table 1, column 1 for the G530 mutants and line 1 for the A1492 mutants). The ribosomes mutated at A1492 all expressed very similar enzyme activities, $1.5 \%-2 \%$ the wild-type level. The ribosomes mutated at G530 showed much more variation, with G530U and G530 $\Delta$ ribosomes producing more activity than either G530A or G530C. In fact, the latter two mutant ribosomes produced significantly less enzyme activity than did cells lacking any dedicated ribosomes; G530A and G530C ribosomes expressed $1.9 \pm 0.1$ and $4.1 \pm 0.4$ units, respectively, compared with the $10 \pm 0.9$ units in the absence of dedicated ribosomes. This result suggests that, rather than simply failing to translate the mRNA, these ribosomes may actually block the low efficiency translation of the mRNA by endogenous ribosomes, perhaps by the mutant ribosomes stalling on the mRNA and precluding the normally infrequent passage of translationally competent wild-type ribosomes.

The enzyme activity produced may not directly correlate to the amount of protein produced. For example, Hughes (1991) demonstrated that error-prone EF-1A mutants of Salmonella typhimurium produce less $\beta$-galactosidase activity in vivo than wild type, despite producing equivalent amounts of the protein. Apparently, multiple missense errors during translation of the protein tend to impair enzyme function and thus reduce specific activity. The reduction ranged from $46 \%$ to $67 \%$ of wild type depending on the mutant tested. We have not excluded the idea that some of the reduction in activity in our data may result from misincorporation.

\section{Multiply mutant ribosomes show no compensatory ability to rescue ribosome activity}

These data confirm the work of others (Santer et al. 1993) suggesting that G530 and A1492 mutations strongly reduce ribosome activity. As shown in Figure 1A, the structure of bacterial ribosome containing a cognate codon-anticodon complex shows that G530 and A1492 pair through an N1$\mathrm{H} \cdots \mathrm{N} 1$ hydrogen bond and that they interact with the middle codon-anticodon base pair with their $2^{\prime}-\mathrm{OH}$ groups (Ogle et al. 2001). The fact that replacing the cognate interaction with a near-cognate interaction disrupts these interactions between the two residues (Ogle et al. 2001) suggests that the nucleotides play a role in cognate recognition. Based on molecular modeling, Sanbonmatsu and Joseph (2003) predicted that exchanging the A and G would not disrupt any of these hydrogen bonds and that a double mutant, G530A A1492G, would allow codon recognition, and would show significant ribosomal activity. We tested this hypothesis by constructing a pDT10 plasmid with the desired double mutation using QuikChange mutagenesis. Table 1 shows that translation of $\beta$-galactosidase in the single mutants G530A (1.9 \pm 0.1 units) and A1492G (32 \pm 2.9 units) increased slightly in the double mutant G530A A1492G (37 \pm 0.9 units). This increase is far less than the restoration predicted by Sanbonmatsu and Joseph (2003), so exchanging G530 and A1492 does not restore significant ribosomal activity.

We extended this work by constructing the full set of 16 double mutants (G530A, G530C, G530U, and G530د each with A1492C, A1492G, A1492U, or A1492A) and found that none of the double mutants produces more than a very low level of lac $Z$ translation (Table 1). We conclude that only the combination of G530 and A1492 provides substantial ribosomal activity and that no other pair of nucleotides can fully replace them in the ribosome's accuracy center. It is true, though, that each of the A1492 mutations tested reversed the apparent dominant negative effect of the G530A and G530C mutations. It could be that the double 

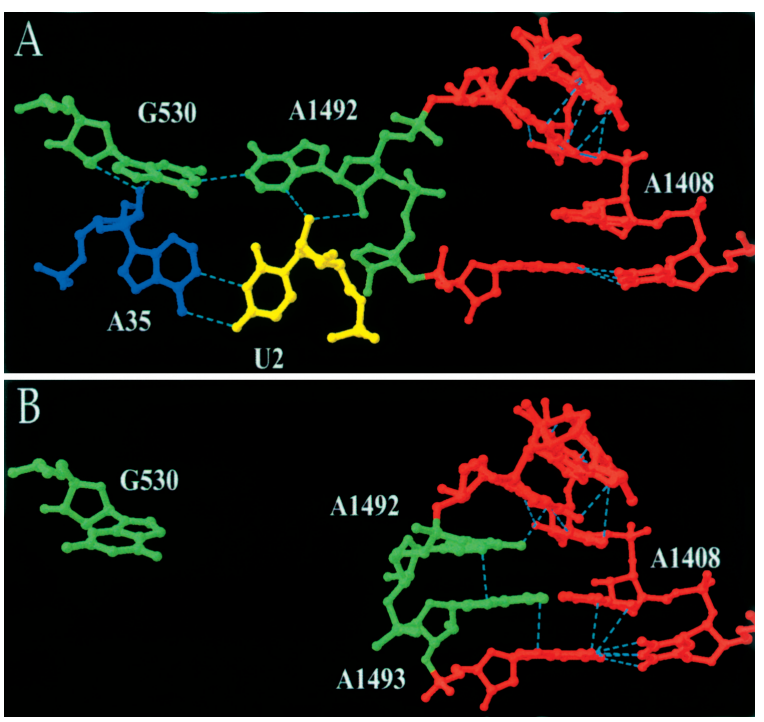

FIGURE 1. Structures of A-site nucleotides in the Thermus thermophilus bacterial ribosome. (A) Structure of the A site bound by a cognate tRNA anticodon loop (Ogle et al. 2001). The figure shows the $\mathrm{N} 1-\mathrm{H} \cdots \mathrm{N} 1$ hydrogen bond between bases G530 and A1492 and the hydrogen bonding to the 2 '-OH groups of the middle base pair of the codon anticodon complex from the $2^{\prime}-\mathrm{OH}$ and N3 positions of both G530 and A1492. The base of A1492 has been removed for clarity; it is located in front of nucleotide $\mathrm{U} 2$ of the codon in this view. (B) Structure of an empty A site (Wimberly et al. 2000). Note that G530, A1492, and A1493 have rotated away from the A site. G530 has rotated from the anti to the syn conformation, and the entire A1492 and A1493 nucleotides have rotated to stack within Helix 44, part of which is shown on the right of the figure. A1408 is shown interacting with A1493. Sanbonmatsu and Joseph (2003) proposed that G1492 would replace A1493 to make a more stable G1492-A1408 pair. The figures were created using coordinates from PDB files 1IBM and 1J5E from the Research Collaboratory for Structural Bioinformatics database using Swiss-PdbViewer (Guex and Peitsch 1997).

mutation reverses the dominance by making the ribosomes nonfunctional and therefore unable to block translation by the endogenous ribosomes. This is unlikely, however, because the translation level by these double mutant ribosomes is significantly higher than that in the absence of the dedicated ribosomes. We suspect, therefore, that the combination of mutations restores a low level of translational activity to the G530A and G530C mutant ribosomes.

\section{Changes to rRNA nucleotide A1408 significantly improve translation activity of minority ribosomes mutated at G530 and A1492}

When a cognate codon occupies the A site, A1492 and A1493 flip from a position stacked within Helix 44 of the 30 S subunit (the "open" conformation shown in Fig. 1B) to a position extending away from Helix 44 and toward the codon-anticodon complex (the "closed" conformation shown in Fig. 1A) (Ogle et al. 2001). This change allows the nucleotides to engage the codon-anticodon complex. The same structure forms in the presence of the error-inducing antibiotic paromomycin. The antibiotic binds to a pocket in Helix 44 causing A1492/A1493 to adopt the closed conformation in the absence of the codon (Carter et al. 2000). By forcing the adoption of the closed complex, the antibiotic may decrease the energetic cost associated with an incorrect tRNA binding to the A site (Ogle et al. 2003). Sanbonmatsu and Joseph (2003) noted a second interaction may occur within the A1492G mutant ribosome by the formation of a N1-H...N1 hydrogen bond between G1492 and A1408; no such bond could form with A1492. This hydrogen bond, they argue, might stabilize the open conformation by increasing the energetic cost to flipping G1492 and A1493 out of Helix 44. This extra cost might reduce the activity of the G530A A1492G double mutant ribosomes. Sanbonmatsu and Joseph (2003) suggested that mutating A1408 to block pairing with G1492 might increase the activity of the G530A A1492G ribosomes.

We tested this hypothesis by constructing a saturating set of triple mutations combining single and double mutations of G530 and A1492 with all possible mutations of A1408 (all four possible changes including the 1 nt deletion). We measured the relative translation activity of each of the mutant ribosomes using the lac $Z$ reporter as above.

As shown in Table 2, combining mutations at A1408 with the A1492 mutations had little effect on translation activity, although in nearly every case the A1408/A1492 double mutants have less activity than the corresponding A1492 single mutant. Combining the A1408 mutations with those at G530 had a very different effect; in most cases, the double mutants had much higher activity than the corresponding G530 mutant. The G530A and G530C mutations are an extreme example. Combining any A1408 mutation (A1408C, A1408G, A1408U, or A1408D) increased the activity of these two mutants substantially. Two of these double mutants had unusually high activity. Activity of the G530C A1408C double mutant was 32 times higher than that of the G530C single mutation, and activity of G530A A1408C was 37 times higher than of G530A. The absolute activity of these mutant ribosomes was, however, still very much lower than wild type, with G530C A1408C mutant ribosomes having only $6.5 \%$ the activity of wild type and G530A A1408C mutants, only 3.5\%. The only other G530 mutants showing a similar interaction are G530U A1408s and G530U A1408U, with $6 \%$ and $4.5 \%$ the wild-type activity. The rest of the G530 mutants had an average activity of $2 \%( \pm 0.8 \mathrm{SD})$ of the wild type.

The effect of A1408 mutations on the activity of the G530 A1492 double mutations overall showed little effect, with increases and decreases in roughly similar numbers. There were, however, several exceptions. Three triple mutants had significantly higher activity: G530A/A1408G/ A1492G had 4.5\% of wild-type activity, G530U/A1408G/ A1492C had 4\%, and G530د/A1408U/A1492U had 5.5\%. A single triple mutant had extremely low activity. The

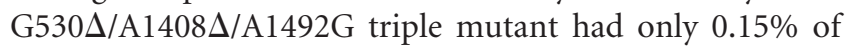


TABLE 2. Expression of lacZ under control of G530/A1408/A1492 triple mutant ribosomes

\begin{tabular}{|c|c|c|c|c|c|c|}
\hline \multirow[b]{2}{*}{530} & \multicolumn{5}{|c|}{1492} & \multirow[b]{2}{*}{1408} \\
\hline & A & $\mathrm{C}$ & G & $U$ & $\Delta$ & \\
\hline \multirow[t]{5}{*}{ G } & $2000 \pm 38$ & $41 \pm 0.8$ & $32 \pm 2.9$ & $42 \pm 3.4$ & $27 \pm 3.5$ & A \\
\hline & $1800 \pm 38$ & $19 \pm 1.1$ & $11 \pm 0.4$ & $34 \pm 1.1$ & $23 \pm 1.7$ & $\mathrm{C}$ \\
\hline & $1000 \pm 65$ & $12 \pm 0.3$ & $18 \pm 0.4$ & $32 \pm 1.3$ & $55 \pm 2.3$ & G \\
\hline & $130 \pm 9.5$ & $30 \pm 1.4$ & $29 \pm 2.1$ & $28 \pm 1.9$ & $29 \pm 0.5$ & U \\
\hline & $120 \pm 14$ & $47 \pm 1.1$ & $27 \pm 2.1$ & $22 \pm 0.9$ & $29 \pm 0.5$ & $\Delta$ \\
\hline \multirow[t]{5}{*}{ A } & $1.9 \pm 0.1$ & $22 \pm 1.5$ & $37 \pm 0.9$ & $28 \pm 0.4$ & $22 \pm 1.0$ & A \\
\hline & $70 \pm 3.4$ & $19 \pm 1.1$ & $38 \pm 2.9$ & $42 \pm 2.0$ & $48 \pm 2.9$ & $\mathrm{C}$ \\
\hline & $55 \pm 1.9$ & $18 \pm 0$ & $91 \pm 5.5$ & $41 \pm 1.3$ & $22 \pm 0.6$ & G \\
\hline & $40 \pm 3.0$ & $52 \pm 2.6$ & $52 \pm 1.1$ & $37 \pm 0.6$ & $40 \pm 2.6$ & U \\
\hline & $54 \pm 1.1$ & $52 \pm 0.8$ & $58 \pm 1.5$ & $58 \pm 2.5$ & $22 \pm 0.8$ & $\Delta$ \\
\hline \multirow[t]{5}{*}{ C } & $4.1 \pm 0.4$ & $50 \pm 0.9$ & $17 \pm 0.6$ & ND & ND & A \\
\hline & $130 \pm 14$ & $24 \pm 1.2$ & $57 \pm 1.6$ & $51 \pm 2.1$ & $30 \pm 0.6$ & $\mathrm{C}$ \\
\hline & $31 \pm 1.7$ & $25 \pm 1.3$ & $25 \pm 0.8$ & $31 \pm 1.1$ & $21 \pm 0.8$ & G \\
\hline & $50 \pm 3.5$ & $21 \pm 0.7$ & $24 \pm 1.6$ & $51 \pm 4.3$ & $35 \pm 1.3$ & U \\
\hline & $43 \pm 3.5$ & $50 \pm 3.0$ & $33 \pm 1.0$ & $47 \pm 1.4$ & $27 \pm 0.9$ & $\Delta$ \\
\hline \multirow[t]{5}{*}{$U$} & $62 \pm 1.6$ & $48 \pm 1.3$ & $44 \pm 2.2$ & $50 \pm 4.0$ & ND & A \\
\hline & $66 \pm 2.7$ & $27 \pm 3.8$ & $23 \pm 0.5$ & $30 \pm 0.4$ & $24 \pm 0.8$ & $\mathrm{C}$ \\
\hline & $36 \pm 1.1$ & $79 \pm 2.4$ & $26 \pm 3.1$ & ND & $43 \pm 1.7$ & G \\
\hline & $89 \pm 6.5$ & $45 \pm 1.5$ & $22 \pm 0.8$ & $48 \pm 2.0$ & $47 \pm 1.8$ & U \\
\hline & $120 \pm 10.0$ & $48 \pm 2.3$ & $3.1 \pm 0.3$ & $52 \pm 2.2$ & $33 \pm 2.1$ & $\Delta$ \\
\hline \multirow[t]{5}{*}{$\Delta$} & $17 \pm 0.4$ & ND & $11 \pm 0.3$ & $74 \pm 2.9$ & $70 \pm 2.8$ & A \\
\hline & $15 \pm 0.3$ & ND & $61 \pm 2.0$ & $50 \pm 1.2$ & $23 \pm 0.5$ & C \\
\hline & $14 \pm 0.4$ & ND & ND & $48 \pm 3.0$ & $65 \pm 3.6$ & G \\
\hline & $46 \pm 0.6$ & $76 \pm 2.7$ & $52 \pm 1.8$ & $110 \pm 4.3$ & $60 \pm 1.8$ & $U$ \\
\hline & $19 \pm 0.6$ & ND & $30 \pm 0.9$ & $25 \pm 0.5$ & $72 \pm 1.8$ & $\Delta$ \\
\hline
\end{tabular}

$\mathrm{ND}$, not determined.

wild-type activity, far below the average for these triple mutants of $2 \%( \pm 1 \% \mathrm{SD})$ of wild type.

These results are not explicitly consistent with the predictions of Sanbonmatsu and Joseph (2003) but do show that some combinations of mutations at G530, A1408, and A1492 have low, but significant, translational activity.

\section{Mutant rRNAs are incorporated efficiently into ribosomal particles}

The low in vivo activity associated with the rRNA mutations could result from a loss of intrinsic translational activity or it could result from failure to incorporate the mutant rRNA during biogenesis of the $30 \mathrm{~S}$ ribosomal particles. To determine whether the mutations have a biogenesis defect, we tested the abundance of wild type and mutant dedicated $16 \mathrm{~S}$ rRNA to the abundance of endogenous wild-type ribosomes. We purified ribosomal particles as described in Materials and Methods. The purified ribosomes we subjected to quantitative real-time PCR (Q-RT-PCR) reactions using a cDNA copy of the $16 \mathrm{~S}$ rRNA as a template (see Materials and Methods). In the dedicated ribosome system, the anti-SD sequence, nucleotides $1535-1540$ of $16 \mathrm{~S}$ rRNA (5'-CCUCCU-3'), was replaced by an alternative SD sequence (5'-GGGAUU-3'). This difference allowed the design of PCR primers specific for the wild-type or dedicated ribosomal 16S rRNA. Test PCR reactions with the two primers demonstrated that each primer primed synthesis only from the intended primer site (data not shown), indicating that the amount of product produced should reflect the relative abundance of each type of $16 \mathrm{~S}$ rRNA.

Q-RT-PCR assays were performed on the wild-type form of the dedicated 16S rRNA and on the single mutants G530A and A1492G, the double mutant G530A/A1492G, and on triple mutants combining G530A and A1492G with all four changes to A1408 (to U, C, G, and the one-base deletion). Each strain transformed with a version of the pDT10 plasmid expressing the $16 \mathrm{~S}$ rRNA with an alternative SD sequence expressing the modified rRNA as well as the endogenous wild type. We attempted to quantify the amount of each dedicated 16S rRNA as compared with the endogenous molecule. Analysis of salt-washed ribosomes derived from each of the strains showed that the average threshold cycle, the PCR cycle at which the product first shows a signal above background, showed little difference between the endogenous rRNA and any of the dedicated rRNAs tested (see Table 3). There was no difference in the 
TABLE 3. Mutant rRNAs are efficiently incorporated into ribosomal particles

\begin{tabular}{|c|c|c|c|c|}
\hline \multicolumn{3}{|c|}{$16 \mathrm{~S}$ rRNA construct } & \multicolumn{2}{|c|}{ Average threshold cycle $( \pm$ SEM) } \\
\hline 530 & 1408 & 1492 & Endogenous RNA & Specialized rRNA \\
\hline G & A & A & $20 \pm 0.7$ & $20 \pm 1.0$ \\
\hline$\underline{A}$ & A & A & $19 \pm 0.8$ & $17 \pm 1.0$ \\
\hline$\overline{\mathrm{G}}$ & A & $\underline{\mathrm{G}}$ & $18 \pm 0.8$ & $21 \pm 1.4$ \\
\hline$\underline{A}$ & A & $\overline{\mathrm{G}}$ & $20 \pm 0.7$ & $21 \pm 1.7$ \\
\hline$\underline{\bar{A}}$ & $\underline{C}$ & $\underline{\bar{G}}$ & $17 \pm 0.4$ & $19 \pm 0.8$ \\
\hline$\underline{\bar{A}}$ & $\underline{\bar{G}}$ & $\underline{\bar{G}}$ & $16 \pm 0.4$ & $20 \pm 1.7$ \\
\hline$\underline{\bar{A}}$ & $\underline{\bar{U}}$ & $\underline{\bar{G}}$ & $17 \pm 0.5$ & $18 \pm 0.7$ \\
\hline$\underline{\bar{A}}$ & $\underline{\Delta}$ & $\underline{\bar{G}}$ & $17 \pm 0.5$ & $18 \pm 1.8$ \\
\hline
\end{tabular}

block its flipping out to interact with A530. Our data are also incompatible with this hypothesis. We found that combining A1492G with any A1408 mutation failed to rescue activity and in fact caused a small decrease in activity. The effect of the A1408 mutations on the G530A A1492G double mutation did not restore, as predicted, substantial activity.

Among the many triple mutations at G530, A1492, and A1408 tested, a small subset resulted in significantly more ribosome activity than the others. The median activity of all of the double, threshold cycle between the endogenous and wild-type dedicated rRNAs, but for the mutant dedicated rRNAs, the average threshold cycle was 1.4 cycles less than for the endogenous rRNA, which indicates that the mutant dedicated ribosomes were on average $\sim 2$.6-fold lower in concentration than the endogenous ribosomes. This difference was too small to explain the difference in activity between the wild-type and mutant ribosomes, which implies that the lower activity of the mutants must reflect some defect in the process of translation.

\section{DISCUSSION}

The rRNA nucleotides G530 and A1492 are essential to ribosome function. Ribosomes with any change at these positions have extremely low activity. Though the two nucleotides recognize the cognate codon-anticodon complexes, the interactions involve hydrogen bonding by rRNA $2^{\prime}-\mathrm{OH}$ group and are therefore not sequence specific. The spacing of these groups, however, allows G530-A1492 to specifically recognize a correct complex because it corresponds precisely to the dimensions of the target groups in a Watson-Crick base pair. No Watson-Crick or non-WatsonCrick base pair has the 2'-OH groups at precisely the same spacing (Westhof and Fritsch 2000), so no other pair of nucleotides should be able to perform this function.

Sanbonmatsu and Joseph (2003) noted that the reciprocal pair, A530-G1492, could make the same contacts, and therefore ribosomes with this nucleotide pair might have wild-type activity. The data presented here demonstrate that this is not true. The activity of double mutant ribosomes (G530A/A1492G) is not substantially greater than the activity of the more active of the single mutant ribosomes, the A1492G mutants.

When a cognate tRNA occupies the A site, a conformational shift of A1492, flipping it out of its position within Helix 44, allows it to interact with G530 and the codon. anticodon complex in the A site. Sanbonmatsu and Joseph (2003) predicted that G1492 might hydrogen bond with A1408, stabilizing it within Helix 44. In the G530A/A1492G double mutant, this hydrogen bond, they argued, might single or triple mutant ribosomes is $1.9 \%( \pm 1.1 \% \mathrm{SD})$ of wild-type ribosomes. Three multiple mutant ribosomes have substantially higher activity, from $5.5 \%$ to $6 \%$ of wild type, and four others have activities in the range of 3.5\%$4.5 \%$ of wild type. On the other hand, one triple mutant ribosome has much lower than average activity; G530U/ A1408 $\Delta / A 1492 \mathrm{G}$ ribosomes have only $0.15 \%$ of wild-type activity. This level is below that of cells lacking any dedicated ribosome, implying that like G530A and G530C this triple mutant has a dominant negative effect. We cannot, however, explain the activity of these mutations by hypothesizing the formation of paired structures in the A site or within Helix 44. We note, furthermore, that although these mutant ribosomes have the greatest activity among the mutants tested, they remain very much less active than wild-type ribosomes. The fact that no combination of nucleotides tested functions nearly as well as the triplet of G530, A1492, and A1408 seems surprising since G530 and A1492 make no sequence-specific contacts with the codon-anticodon complex. A1492, however, does make sequence specific contacts with other ribosome components. For example, the $N^{6}$ amino group of A1492 hydrogen bonds with the side-chain hydroxyl of rpS12 Ser50 and with the 2'-OH of C518. Replacing A1492 with a guanosine would eliminate these interactions, and, if they were necessary to stabilize the closed conformation, their loss might explain the lack of activity of mutants involving G530A/A1492G.

The assay we have used measures the amount of $\beta$-galactosidase activity produced in vivo by the tested ribosomes. The loss of activity could result from a variety of causes. Because the tested nucleotides directly contact the codon-anticodon helix in the ribosomal A site, altering them might reduce the rate of aminoacyl-tRNA recruitment and thus the amount of protein produced. Alternatively, the mutations might increase the frequency of translational errors. Hughes (1991) showed that increasing in vivo translation errors approximately fourfold in the presence of error-prone EF-1A caused an approximately twofold decrease in $\beta$-galactosidase activity. This reduction results not from a reduction in bulk synthesis but presumably from multiple amino acid misincorporations that result in 
an inactive protein product. We have not explicitly excluded that the mutant ribosomes produce near-normal amounts of protein with extremely low specific activity. However, even were that true it would still be inconsistent with the prediction that G530 and A1492 could be exchanged without affecting ribosomal function.

As an alternative, it may be that Sanbonmatsu and Joseph (2003) were correct that mutant ribosomes with reciprocal exchanges of G530 and A1492 function normally in tRNA recognition but that the mutations disrupt some process other than cognate tRNA recognition during elongation. The three conserved nucleotides might function in other A-site events, including initiation, the translocation step of elongation, or termination. As an example, initiation factor 1 (IF-1) binds in the A site during initiation and, along with rpS12, buries both A1492 and A1493 in protein pockets (Carter et al. 2001). Bulkier guanosine residues could not bind in these pockets, which would explain the loss of function of A1492G and A1493G mutations (Dahlquist and Puglisi 2000; Carter et al. 2001). Thus, the mutant ribosomes tested may lack activity because they disrupt any of multiple interactions with trans-acting factors. The approach of focusing on a single molecular complex underestimates the constraints under which the nucleotides must function in the ribosome. The extreme conservation of critical residues in the ribosome may result from constraint by multiple molecular interactions, not simply during a particular step in translation but during multiple steps and involving multiple partners.

\section{MATERIALS AND METHODS}

\section{Strains}

The E. coli strains used were DH5 $\alpha\left(\mathrm{F}^{-}\right.$Ф80dlacZDM15 $\Delta[$ lacZYA$\operatorname{argF}$ UU169 deoR recAl endA1 hsdR17 $\left[\mathrm{rk}^{-} \mathrm{mk}^{+}\right]$phoA supE44 $\lambda^{-}$ thi-1 gyrA96 relA1) (Hanahan 1983), BW23474 ( $\mathrm{F}^{-} \Delta$ [argF-lac $] 169$ uidA4[del]::pir-116 recA1 rpoS396[Am] endA9[del-ins]::RT rph-1 $h s d R 514$ creC510 robAl) (Haldimann and Wanner 2001), and DT1 (F' ${ }^{-}$\$80dlacZDM15 $\Delta$ [lacZYA-argF]U169 attB::lacZ-SSD deoR recA1 endA1 hsdR17 $\left[\mathrm{rk}^{-} \mathrm{mk}^{+}\right]$phoA phoA supE44 $\lambda^{-}$thi-1 gyrA96 relA1). The construction of DT1 is described below. Bacterial transformation was performed using DH5 $\alpha$ or DT1 strains made chemically competent using the Z-competent protocol (Zymo Research).

\section{Construction of an integrated lacZ gene with alternative Shine-Dalgarno site}

We used the CRIM plasmid integration system described by Halidmann and Wanner (2001) to integrate a modified lacZ reporter into the E. coli chromosome. The plasmid pLA2 has lacZ under the control of the $a r a B$ promoter and expresses kanamycin resistance. It also contains the bacteriophage $\lambda$ attP attachment site, which directs plasmid integration at the attB locus on the E. coli chromosome. A temperature-sensitive helper plasmid pINT-ts encodes the $\lambda$-specific integrase gene, int, which catalyzes the integration event. pLA2 employs the $o r i R_{\gamma}$ replication origin. This origin is derived from the plasmid R6K, and its replication requires the pir gene product (Haldimann and Wanner 2001); it replicates to high copy numbers in the strain BW23474. To test for single-copy integration, colony PCR is performed with four primers, P1, P2, P3, and P4, as described (Haldimann and Wanner 2001), corresponding to the attB site (the P1-P4 fragment), the attL (P1-P2) site, and the attR (P3-P4) site. Successful integration results in the loss of the attP site and creation of $a t t L$ and $a t t R$, while multiple tandem integration will result in maintenance of both the attP and attB site.

QuikChange site-directed mutagenesis using PfuUltra (Stratagene) inserted an alternative ribosome binding sequence, $5^{\prime}$ ATCCCT-3', six base pairs upstream of the lac $Z$ start codon of pLA2. After mutation by DNA sequencing was confirmed, the plasmid was digested with NdeI and DraIII (New England Biolabs), and the fragment containing the mutated $\operatorname{lac} Z$ was ligated into a similarly digested wild-type pLA2 to create the plasmid pDT118. The structure of pDT118 was confirmed by DNA sequencing and it was integrated into the chromosome of the E. coli strain DH5 $\alpha$ as described by Halidmann and Wanner (2001). Putative integrants were then tested for successful integration through selective plating and colony PCR. The resulting strain was called DT1.

\section{Construction of a plasmid expressing mutant $16 S$ rRNA}

The plasmid pRNA122 (Lee et al. 1996, 1997, 2001) expresses the rrnB operon (which contains the 16S, 23S, and 5S rDNA) under the control of the lacUV5 promoter controlled by $l a c I^{\mathrm{q}}$ (a lac repressor overexpressing mutation), the gene for chloramphenicol acetyl transferase (cam) under constitutive transcription control of a mutant $\operatorname{trp}$ promoter, and the bla gene encoding ampicillin resistance. Digestion of pRNA122 using the restriction enzymes $\mathrm{XbaI}$ and KpnI generated a 4.4-kbp fragment containing the $16 \mathrm{~S}$

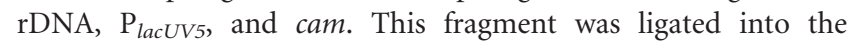
multicopy cloning vector Litmus28 (New England Biolabs) using the same enzymes to create the plasmid pDT10. QuikChange sitedirected mutagenesis of this plasmid using PfuUltra (Stratagene) was used to mutate G530, A1408, and A1492 using specific oligonucleotides. The structure of each of the mutated 16S rRNA genes was verified by DNA sequencing.

\section{$\boldsymbol{\beta}$-Galactosidase assay}

The lacZ-SSD reporter strain DT1 was transformed with the dedicated 16S rRNA expressing plasmid pDT10 or one of its mutant derivatives. Transformed clones were inoculated and grown overnight in LB-AI medium supplemented with $1 \mathrm{mM}$ arabinose. This level of arabinose fully induces the transcription of $16 \mathrm{~S}$ rRNA from pDT10. These cultures were then diluted 100-fold into the same medium and grown for $3 \mathrm{~h}$ or until the $\mathrm{OD}_{600}$ was between 0.28 and 0.7 . The cells were then permeabilized and assayed for $\beta$-galactosidase activity as described previously (Miller 1972). All assays were performed on at least three independent transformants and in triplicate.

\section{Ribosome purification and quantitative real-time PCR assays}

Total cell RNA was prepared from E. coli strains transformed with plasmids encoding specialized ribosomes. Transformants were 
grown to an $\mathrm{OD}_{600}$ of $\sim 1.0$ in LB-AI medium. Ribosomal RNA (rRNA) was purified using the RNeasy Mini Kit (Qiagen) from salt-washed cellular ribosomes that had been purified essentially as described (Zengel et al. 2003).

These RNA preparations were subjected to digestion with RNase-free DNase I (Fermentas) for $30 \mathrm{~min}$ (in $10 \mathrm{mM}$ Tris, $\mathrm{pH}$ 7.5, $2.5 \mathrm{mM} \mathrm{MgCl}$, and $0.1 \mathrm{mM} \mathrm{CaCl}_{2}$ ), which ensured removal of any plasmid DNA. After digestion, addition of $2.5 \mathrm{mM}$ EDTA and incubation at $65^{\circ} \mathrm{C}$ for 15 min inactivated the enzyme. The resulting RNA preparation was used as a template for synthesis of cDNA using primers specific for the endogenous wild type $16 \mathrm{~S}$ rRNA $\left(5^{\prime}\right.$-TTTAACTTCTCAAAC- $\left.3^{\prime}\right)$ or the specialized $16 \mathrm{~S}$ rRNA (5'-TAATCCCATGATCCA-3') using the First Strand cDNA Synthesis Kit (Fermentas) according to the manufacturer's directions.

Q-RT-PCR was performed using a SYBR Green quantitative PCR kit (Molecular Probes). Primers for the endogenous 16S rRNA (5' -ATTCCTCCACTAGGTTGGC-3') or for the specialized $16 \mathrm{~S}$ rRNA (5'-TAATCCCATGATCCA-3') were used in the PCR reaction in conjunction with a common second primer $\left(5^{\prime}\right.$ AGGTCGCTTCTCTTTGTA-3'). The protocol for the PCR was a single step of $95^{\circ} \mathrm{C}$ for $1.5 \mathrm{~min}$ followed by 40 cycles of $95^{\circ} \mathrm{C}(20$ $\mathrm{sec}), 60^{\circ} \mathrm{C}(20 \mathrm{sec})$, and $72^{\circ} \mathrm{C}(20 \mathrm{sec})$. The Q-RT-PCR reactions were performed on an iCycler IQ thermocycler (Bio-Rad).

\section{ACKNOWLEDGMENTS}

We thank Belinda Jackson for technical assistance with Q-RT-PCR and Sephorah Zaman for technical assistance with purification of salt-washed ribosomes. A grant from the National Institutes of Health (R01 GM029480) to P.J.F. supported this work. D.L.T. was supported by a Ruth L. Kirschstein National Research Service Award from the National Institutes of Health (F31 GM073575).

Received March 9, 2007; accepted May 18, 2007.

\section{REFERENCES}

Abdi, N.M. and Fredrick, K. 2005. Contribution of 16S rRNA nucleotides forming the $30 \mathrm{~S}$ subunit $\mathrm{A}$ and $\mathrm{P}$ sites to translation in Escherichia coli. RNA 11: 1624-1632.

Carter, A.P., Clemons, W.M., Brodersen, D.E., Morgan-Warren, R.J., Wimberly, B.T., and Ramakrishnan, V. 2000. Functional insights from the structure of the $30 \mathrm{~S}$ ribosomal subunit and its interactions with antibiotics. Nature 407: 340-348.

Carter, A.P., Clemons Jr., W.M., Brodersen, D.E., Morgan-Warren, R.J., Hartsch, T., Wimberly, B.T., and Ramakrishnan, V. 2001. Crystal structure of an initiation factor bound to the $30 \mathrm{~S}$ ribosomal subunit. Science 291: 498-501.

Dahlquist, K.D. and Puglisi, J.D. 2000. Interaction of translation initiation factor IF1 with the E. coli ribosomal A site. J. Mol. Biol. 299: $1-15$.

Gromadski, K.B. and Rodnina, M.V. 2004. Kinetic determinants of high-fidelity tRNA discrimination on the ribosome. Mol. Cell 13: 191-200.

Guex, N. and Peitsch, M.C. 1997. SWISS-MODEL and the SwissPdbViewer: An environment for comparative protein modeling. Electrophoresis 18: 2714-2723.

Haldimann, A. and Wanner, B.L. 2001. Conditional-replication, integration, excision, and retrieval plasmid-host systems for gene structure-function studies of bacteria. J. Bacteriol. 183: 6384-6393.

Hanahan, D. 1983. Studies on transformation of Escherichia coli with plasmids. J. Mol. Biol. 166: 557-580.
Hopfield, J. 1974. Kinetic proofreading: A new mechanism for reducing errors in biosynthetic processes requiring high specificity. Proc. Natl. Acad. Sci. 71: 4135-4139.

Hughes, D. 1991. Error-prone EF-Tu reduces in vivo enzyme activity and cellular growth rate. Mol. Microbiol. 5: 623-630.

Karplus, M. and McCammon, J.A. 2002. Molecular dynamics simulations of biomolecules. Nat. Struct. Biol. 9: 646-652.

Lee, K., Holland-Staley, C.A., and Cunningham, P.R. 1996. Genetic analysis of the Shine-Dalgarno interaction: Selection of alternative functional mRNA-rRNA combinations. RNA 2: 1270-1285.

Lee, K., Holland-Staley, C.A., and Cunningham, P.R. 2001. Genetic approaches to studying protein synthesis: Effects of mutations at Psi516 and A535 in Escherichia coli 16S rRNA. J. Nutr. 131: 2994S3004S.

Lee, K., Varma, S., SantaLucia Jr., J., and Cunningham, P.R. 1997. In vivo determination of RNA structure-function relationships: Analysis of the 790 loop in ribosomal RNA. J. Mol. Biol. 269: 732-743.

Lim, V.I. and Curran, J.F. 2001. Analysis of codon:anticodon interactions within the ribosome provides new insights into codon reading and the genetic code structure. RNA 7: 942-957.

Miller, J.H. 1972. Experiments in molecular genetics. Cold Spring Harbor Laboratory, Cold Spring Harbor, NY.

Ninio, J. 1975. Kinetic amplification of enzyme discrimination. Biochimie 57: 587-595.

Ogle, J., Brodersen, D., Clemons Jr., W.M., Tarry, M., Carter, A., and Ramakrishnan, V. 2001. Recognition of cognate transfer RNA by the 30S ribosomal subunit. Science 292: 897-902.

Ogle, J.M., Carter, A.P., and Ramakrishnan, V. 2003. Insights into the decoding mechanism from recent ribosome structures. Trends Biochem. Sci. 28: 259-266.

Ogle, J.M., Murphy, F.V., Tarry, M.J., and Ramakrishnan, V. 2002. Selection of tRNA by the ribosome requires a transition from an open to a closed form. Cell 111: 721-732.

Powers, T. and Noller, H.F. 1990. Dominant lethal mutations in a conserved loop in 16S rRNA. Proc. Natl. Acad. Sci. 87: 1042-1046.

Rodnina, M.V., Gromadski, K.B., Kothe, U., and Wieden, H.J. 2005. Recognition and selection of tRNA in translation. FEBS Lett. 579: 938-942.

Sanbonmatsu, K.Y. and Joseph, S. 2003. Understanding discrimination by the ribosome: Stability testing and groove measurement of codon-anticodon pairs. J. Mol. Biol. 328: 33-47.

Santer, M., Santer, U., Nurse, K., Bakin, A., Cunningham, P., Zain, M., O'Connell, D., and Ofengand, J. 1993. Functional effects of a $\mathrm{G}$ to $\mathrm{U}$ base change at position 530 in a highly conserved loop of Escherichia coli 16S RNA. Biochemistry 32: 5539-5547.

Shine, J. and Dalgarno, L. 1974. The 3 '-terminal sequence of Escherichia coli $16 \mathrm{~S}$ ribosomal RNA: Complementarity to nonsense triplets and ribosome binding sites. Proc. Natl. Acad. Sci. 71: 13421346.

Simonson, A.B. and Lake, J.A. 2002. The transorientation hypothesis for codon recognition during protein synthesis. Nature 416: 281285.

Thompson, R. 1988. EFTu provides an internal kinetic standard for translational accuracy. Trends Biochem. Sci. 13: 91-93.

VanLoock, M.S., Easterwood, T.R., and Harvey, S.C. 1999. Major groove binding of the tRNA/mRNA complex to the $16 \mathrm{~S}$ ribosomal RNA decoding site. J. Mol. Biol. 285: 2069-2078.

Westhof, E. and Fritsch, V. 2000. RNA folding: Beyond Watson-Crick pairs. Struct. Fold. Des. 8: R55-R65.

Wimberly, B.T., Brodersen, D.E., Clemons Jr., W.M., MorganWarren, R.J., Carter, A.P., Vonrhein, C., Hartsch, T., and Ramakrishnan, V. 2000. Structure of the 30S ribosomal subunit. Nature 407: 327-339.

Yoshizawa, S., Fourmy, D., and Puglisi, J.D. 1999. Recognition of the codon-anticodon helix by ribosomal RNA. Science 285: 1722-1725.

Zengel, J.M., Jerauld, A., Walker, A., Wahl, M.C., and Lindahl, L. 2003. The extended loops of ribosomal proteins L4 and L22 are not required for ribosome assembly or L4-mediated autogenous control. RNA 9: 1188-1197. 

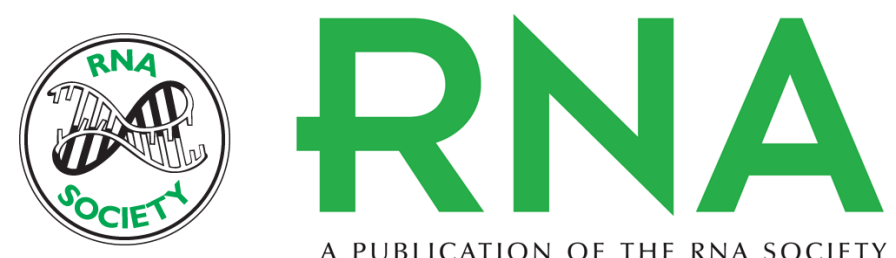

A PUBLICATION OF THE RNA SOCIETY

\section{Testing constraints on rRNA bases that make nonsequence-specific contacts with the codon -anticodon complex in the ribosomal A site}

Dwayne L. Taliaferro and Philip J. Farabaugh

RNA 2007 13: 1279-1286 originally published online June 25, 2007

Access the most recent version at doi:10.1261/rna.552007

$\begin{array}{ll}\text { References } & \begin{array}{l}\text { This article cites } 31 \text { articles, } 11 \text { of which can be accessed free at: } \\ \text { http://rnajournal.cshlp.org/content/13/8/1279.full.html\#ref-list-1 }\end{array}\end{array}$

License

Email Alerting Receive free email alerts when new articles cite this article - sign up in the box at the Service top right corner of the article or click here. 